\title{
Incidence of symptomatic CSF viral escape in HIV infected patients receiving atazanavir/ritonavir (ATV/r)-containing ART: a tertiary care cohort in western India
}

\author{
Atul K. Patel ${ }^{1}$, Ketan K. Patel ${ }^{1}$, Swati Gohel ${ }^{1}$, Ambuj Kumar ${ }^{2}$, and Scott Letendre ${ }^{3}$ \\ ${ }^{1}$ Infectious Diseases Clinic, "VEDANTA" Institute of Medical Sciences, 3rd Floor Navrangpura, \\ Ahmedabad 380009, India \\ ${ }^{2}$ Division and Center for Evidence Based Medicine and Outcomes Research: Department of \\ Internal Medicine, University of South Florida, Tampa, FL 33612, USA \\ ${ }^{3}$ HIV Neurobehavioral Research Center, Antiviral Research Center, University of California, San \\ Diego, 150 West Washington Street, San Diego, CA 92103, USA
}

\begin{abstract}
This single-center study attempts to quantify the incidence of symptomatic CSF viral escape (CSFVE) in patients receiving atazanavir/r (ATV/r)-containing regimen. We performed a retrospective analysis of patients receiving ATV/r-containing ART who were diagnosed with symptomatic CSFVE from August 2012 to January 2017. Primary objective was to assess the incidence of symptomatic CSFVE in patients receiving ATV/r-containing ART in clinical practice. Incidence rates were calculated by dividing the number of patients who experienced CSFVE by the number of person-months at risk and summarized as per 10,000 (ten thousand) person-months at risk. Nine hundred thirty-three patients receiving ATV/r containing ART with a total of 36,068 person-months of follow-up were included. Incidence rate of symptomatic CSFVE was 4.4 per 10,000 person-months (95\% CI 2.7 to 7.2). The incidence of CSFVE was 9.5 per 10,000 personmonths (95\% CI 5.7 to 15.7 ) when the nadir CD4 count was $\leq 200$ compared to 0.49 (95\% CI 0.07 to 3.5) with a nadir CD4 count > 200 (IRR 19.1 (95\% CI 2.93 to 802.8), $p<0.0001$ ). Nadir CD4 count $\leq 200$ was associated with substantially increased risk of symptomatic CSFVE, further strengthening efforts to diagnose and treat patients early in disease.
\end{abstract}

\section{Keywords}

HIV-associated neurocognitive disorders; CSF HIV RNA; CSF HIV escape; CNS penetration of ART

Correspondence to: Atul K. Patel.

Conflict of interest

The authors declare that they have no conflict of interest. 


\section{Background}

Antiretroviral therapy (ART) has led to significant improvements in life expectancy as well as quality of life of people living with HIV infection. Incidence of HIV-associated dementia has markedly reduces with the use of ART. Complete suppression of plasma HIV RNAwith current ART regimen is associated with improved clinical outcome of HIV patients. Despite these tremendous clinical benefits and the overall reduction in the incidence of HIVassociated neurocognitive disorder (HAND), the prevalence of milder forms of HAND remained stable in patients receiving ART over the past two decades and may have even increased. During the past decade, another neurological condition, CSF viral escape (CSFVE), has emerged and can be either neurologically symptomatic or asymptomatic. CSFVE is associated with a HIV-1 HIV RNA that is higher in CSF than in plasma during ART. This is unusual since CSF HIV-1 HIV RNA is typically at least one $\log _{10}$ lower than plasma HIV RNA(Price and Spudich 2008), and it was previously thought that nearly all patients achieve suppression of HIV RNA in both CSF and plasma while receiving ART.

CSFVE during ART appears to occur in three clinical contexts: (1) Asymptomatic CSF escape, (2) Neurosymptomatic CSF escape, and (3) Secondary escape. Patients with asymptomatic CSFVE have low levels of CSF HIV (50-200 copies/ml) with normal CSF examination without neurological symptoms. It may be the CSF equivalent of HIV viral blips in plasma. One study reported $10 \%$ of patients in a cross-sectional cohort study had asymptomatic CSF escape (Eden et al. 2010; Eden et al. 2016). Patients, who developed new or progressive neurological symptoms and had elevated CSF HIV RNA are described under neurosymptomatic CSFVE. This category may be more likely to demonstrate evidence of inflammation in CSF, e.g., pleocytosis. This category may equate to virological failure in the CSF compartment. Secondary escape is described in patients with CNS infections like syphilis, cryptococcal meningitis, or toxoplasmosis causing inflammation and high HIV RNA in CSF (Ferretti et al. 2015). Several published reports have linked ART (e.g., drug class, estimated drug distribution into CSF) or viral (e.g., drug resistance) characteristics to CSFVE (Canestri et al. 2010; Bogoch et al. 2011; Tamarit Mdel et al. 2012; Beguelin et al. 2016). Persistent HIV replication in CNS compartment leads to inflammatory damage to neuronal cells and is associated with neurological symptoms. Several case reports and small case series have described the association between CSF RNA and CNS disease (Cinque et al. 1998; Robertson et al. 1998; Christo et al. 2007). Controversy exists regarding the use of ART with high CPE score and better neurological outcomes. One trial randomized HAND to patients to either a "better penetrating" or "worse penetrating" ART strategy but failed to demonstrate benefit in reducing neurocognitive abnormalities, although the underenrolled trial was underpowered.(Ellis et al. 2014) Several case reports and small case series symptomatic CSF escape have, however, reported rapid clinical response and better neurocognitive outcome with ART modification based on genotypic resistance testing and estimated ART drug distribution into the CNS (Canestri et al. 2010; Peluso et al. 2012; Fabbiani et al. 2015). Symptomatic CSF escape, however, appears to be a rare phenomenon. The incidence of symptomatic CSFVE has not been studied in patients receiving atazanavir/ ritonavir-containing ART. The primary objective of the study was to assess the incidence of symptomatic CSFVE in patients receiving atazanavir/ritonavir (ATV/r)-containing 
antiretroviral treatment in clinical practice. Secondary objectives were to describe the clinical presentation, risk factors, and clinical response to optimized ART.

\section{Methods}

This is a retrospective cohort study of HIV-1 patients receiving atazanavir/r-containing ART who were diagnosed with symptomatic CSFVE between August 2012 and January 2017 at a tertiary care center in western India. CSFVE was defined as detectable CSF HIV RNA with undetectable plasma HIV RNA or CSF RNA $>1 \log _{10}$ copies/mL than plasma HIV RNA. Patients fulfilling criteria for symptomatic CSFVE were included in the study. Patients with concomitant active CNS infection were excluded. Data of all the patients receiving ATV/r containing ARTwere retrieved from clinic database to calculate incidence.

\section{Patient's evaluation for CSF escape}

All HIV-infected patients receiving antiretroviral therapy who present with new onset of neurological abnormalities to the outpatient clinic underwent CSF examination (routine, microscopy, cryptococcal antigen, VDRL), CSF RNA, plasma RNA, and MRI of the brain (whenever clinically indicated) in addition to routine checkup (TSH, B12 estimation, biochemistry and infection work up). Of all patients diagnosed with symptomatic CSF viral escape, only those patients receiving atazanavir/r-contacting regimen were analyzed for this study. Data were collected on demographic details, duration of HIV, duration of ART regimen, clinical presentation, and time to clinical response following CNS penetration effectiveness (CPE) score-optimized ART (version 2010 by Letendre and colleagues). (Letendre et al. 2010) Study patients were followed up clinically at 2, 4, and 12 weeks, while plasma HIV RNA was repeated at 12 weeks for study patients with detectable HIV RNA at the time of the escape.

CSF and plasma HIV-1 RNA tests were carried out by the Abbott Real Time HIV-1 (Abbott Laboratories, Abbott Park, Illinois, USA) assays with lower limit of quantification (LLQ) of 40 copies/ml or Cobas TaqMan HIV-1 assay (Roche), with a LLQ of 20 copies/mL at study site. The same assay was used to test paired blood and CSF HIV RNA. HIV-1 resistance assay from CSF and plasma were done at National AIDS Research Institute (NARI) Pune. CSF ART levels were not measured. Ethics committee approval was taken for this retrospective study.

\section{Statistical methods}

Patient characteristics are reported as proportions (binary data) or means with accompanying 95\% confidence intervals (95\% CI) and standard error. The incidence rates were calculated by dividing the number of patients who experienced CSF escape by the number of personmonths at risk and summarized as per 10,000 (ten thousand) person-months at risk. The follow-up time was measured from ART initiation until the date of CSF escape or the last follow-up visit. We used an intent-to-continue-treatment approach not accounting for lost to follow-up or change in ART regimen. The difference in incidence rates according to factors associated with CSF escape was summarized as incidence rate ratio (IRR) along with 95\% CI. Receiver operating characteristic (ROC) curve analysis was performed to determine the 
CD4 cutoff to identify patients at risk for CSF escape. The area under the curve (AUC) was calculated along with $95 \%$ confidence intervals (CI) for all comparisons. The $p$ value for all comparisons was set at $5 \%$. All the analyses were done using STATA/SPSS statistical analysis software.

\section{Results}

Since the introduction of atazanavir in 2006 in India, 933 HIV-1 patients underwent treatment with atazanavir/r-containing combination ART up to January 2017 at our clinic.

Twenty-six patients were diagnosed with symptomatic CSFVE during the study period receiving different ART regimens of which 16 patients receiving ATV/r-containing regimen were analyzed to characterize risk factors and to calculate incidence. Baseline characteristics of patients receiving ATV/r-containing regimen and diagnosed CSFVE are described in Table 1 and the flow of the study cohort is illustrated in Fig. 1.

TDF/FTC was the most common NRTI backbone used (686, 73.53\%), followed by AZT/3TC $(235,25.19 \%)$ and $\mathrm{ABC} / 3 \mathrm{TC}(12,1.29 \%)$. Consistent with treatment guidelines in India, most patients $(731 ; 78 \%)$ were treatment-experienced before they started ATV/rcontaining ART, while $202(22 \%)$ were treatment-naïve. Prior ART regimens were typically either EFVor NVP in combination with AZT/3TC. The proportion of patients with baseline CD4 count either $>200(n=470 ; 50 \%)$ or $\leq 200(n=463 ; 50 \%)$ were similar. The mean HIV duration prior to current ART was 54.5 months (95\% CI 51.4 to 57.6 months; standard error \pm 1.58 ; median of 49 months), and the mean duration of current ART was 38.74 months (95\% CI 36.52 to 41.0 months; standard error \pm 1.12 ; median of 30 months) with a total of 36,068 person-months of follow- up. Baseline characteristics of patients receiving atazanavir-containing regimen is shown in Table 1.

Except for two patients, all had multiple presenting symptoms. Impaired memory (56.3\%, $n$ $=9)$ and dizziness $(50 \%, n=8)$ being the most common presentation, followed by tremors $(43.8 \%, n=7)$, gait disturbances $(31.3 \%, n=5)$, psychiatric symptoms, and bradykinesia $(18.8 \%, n=3)$. Two patients each $(12.5 \%)$ had bowel-bladder incontinence and slurred speech along with cognitive abnormalities while two presented with convulsions. One patient complained of encircling lower thoracic dermatomal pain as a sole presenting complaint, and one patient had a headache with visual disturbances.

Out of the 16 patients, ten underwent MRI evaluation, three had normal imaging, one had cortical atrophy and six showed varied symmetrical non-enhancing hyperintense lesions on T2-weighted and FLAIR images in periventricular deep white matter, subcortical right temporal white matter, deep matter gray nuclei, pons, midbrain, and cerebellum consistent with HIV encephalitis.

Genotypic resistance testing (GTR) was performed on 7 of the 16 CSF specimens. HIV did not amplify in 2 of the 7 specimens. Two patients had paired CSF-plasma GTR results. All tested patients had HIV-1, subtype C. Paired plasma sample of patients 3 and 4 (Table 2) had a similar PI and RT mutations. CSF HIV GTR results are shown in Table 2. 


\section{Incidence of CSF escape}

Of the 933 patients, 16 experienced CSF escape resulting in incidence rate of 4.4 per 10,000 person-months (95\% CI 2.7 to 7.2). The incidence of CSF escape was not associated with age, sex, weight, or ART status of the patient (naive vs experience). As shown in Table 3, the incidence of CSF escape was 9.5 (95\% CI 5.7 to 15.7) in patients with a baseline CD4 count of $<200$ compared to 0.49 (95\% CI 0.0698 to 3.5 ) in patients with a CD4 count of $\geq 200$ per 10,000 person-months resulting in IRR of 0.05 (95\% CI 0.001 to 0.34 ) which was statistically significant $(p<0.0001)$. None of the patients receiving AZT/3TC or ABC/3TC developed CSF escape, while 16 out of 686 receiving TDF/FTC developed CSF escape and this difference was statistically significant $(p=0.0086)$ (Fig. 2).

As shown in Fig. 3, a baseline CD4 count of $115 / \mathrm{mm}^{3}$ best balanced sensitivity (70\%) and specificity $(75 \%$ ) for having CSFVE (AUC $=74 \%$; 95\% CI 62 to $82 \%$ ).

ART was changed in all patients with CSF escape. TDF/FTC/AZT/lopinavir/ritonavir (LPV/r) was prescribed in six patients; two patients each were prescribed $\mathrm{AZT} / 3 \mathrm{TC} / \mathrm{TDF} / \mathrm{LPV} / \mathrm{r}, \mathrm{AZT} / 3 \mathrm{TC} / \mathrm{LPV} / \mathrm{r}$, and TDF/FTC/LPV/r while one each changed to TDF/FTC/LPV/r/raltegravir (RAL), AZT/3TC/LPV/r/RAL, AZT/3TC/LPV/r/indinavir (IDV), and TDF/FTC/IDV/r. Median CPE score of ART after a change in treatment was 10.5 (7-13).

Patients were followed for a median duration of $14.5(1-48)$ months $(n=16)$. Symptomatic improvement was considered as a marker of response to therapy but repeat CSF HIV RNA was not performed. All patients had rapid neurological improvement after a change in ART with complete recovery within 1 month. The patient with the advanced neurocognitive disease with bladder and bowel incontinence regained bladder control after 8 days of ART change and started walking without support within a month. All patients are in follow-up with controlled disease and symptoms except for three who had a recurrence of similar neurological symptoms when their new ART regimen failed due to nonadherence.

\section{Discussion}

The role of ART drug characteristics in the pathogenesis of CSFVE continues to be debated. When ART suppresses plasma HIV RNA, it also suppresses CSF HIV RNA in more than $90 \%$ of patients irrespective of estimated drug distribution into the CNS (Staprans et al. 1999; Yilmaz et al. 2006; Ferretti et al. 2015). CSFVE is uncommon but, when it occurs, it may be related to low nadir CD4 counts, CNS infections, suboptimal drug adherence, ART drug resistance, or subtherapeutic ART drug concentrations in CSF (Rawson et al. 2012; Fabbiani et al. 2015).

We identified 26 cases of symptomatic CSFVE during the study period of which 16 (61.5\%) were receiving TDF/FTC/ATV/r with CPE score of 6 . Among the other 10, 6 (23.5\%) received TDF/FTC/LPV/r with CPE score of 7 while one each received TDF/FTC/EFV (CPE score 7), TDF/FTC/IDV/r (CPE score 8), TDF/FTC/LPV/r/IDV (CPE score 11), and RAL/darunavir (DRV)/r (CPE score 6). In our clinic, 23 (88.5\%) of the CSFVE patients were on regimens that had CPE score of $\leq 7$, a possible risk factor for CSFVE, and all 
except one patient were receiving TDF/FTC backbone. Published literature so far reported CSFVE in patients receiving different ART combinations with different CPE score (Nightingale et al. 2016) To our knowledge, this is the first study looking at the incidence of symptomatic CSF escape in patients receiving only ATV/r-containing ART in clinical practice. Atazanavir containing-regimen is recommended second-line ART in India after NNRTI containing regimen failure.

The overall incidence of CSFVE in our study was 4.4 (95\% CI 2.7 to 7.2) per 10,000 person-months follows-up in patients receiving ATV/r-containing ART. This risk increases and is significantly higher in patients with nadir CD4 count of $<200 \mathrm{~mm}^{3}(9.5$ (95\% CI 5.7 to 15.7$)$ per 10,000 person-months) which reconfirms the low nadir CD4 count $\left(<200 \mathrm{~mm}^{3}\right)$ as an important risk factor for CSFVE as described previously in other studies (Canestri et al. 2010; Peluso et al. 2012). CSFVE with or without symptoms is observed in patients receiving PI/r monotherapy and double-boosted PI with suppressed plasma HIV RNA (Vernazza et al. 2007; Santos et al. 2013; Antinori et al. 2015; Ferretti et al. 2016). Mono $\mathrm{ATV} / \mathrm{r}$ study described $15 \%$ risk of CSFVE at 24 weeks, and all three patients with escape had suppressed plasma HIV RNA. Another study also described early CSFVE during PI only therapy (Donath et al. 2016). This raises the importance of backbone NRTI in controlling viral replication in CNS compartment (Vernazza et al. 2007). CPE score may not be as important for NRTI as protease inhibitors, as NRTI's main activity depends on intracellular phosphorylation to active compound rather than measured extracellular drug level. Despite this unique property of NRTIs, TDF with the lowest CPE score of 1 among NRTIs can have a possible association with risk of CSFVE. Study from Cusini et al. reported all four patients diagnosed with CSFVE were receiving TDF/FTC backbone, and none of the patients receiving AZT $(n=39)$ or ABC $(n=18)$ developed CSFVE at any given time in the study while in another study out of the 20 patients described, 12 were receiving TDF, 2 were not received any NRTI and 2 each were receiving single NRTI, AZT/3TC, and ABC/3TC NRTI backbone (Cusini et al. 2013; Nightingale et al. 2016). In our study, also none of the patients receiving AZT/3TC or ABC/3TC developed symptomatic CSFVE, suggests a possible association of the use of TDF with the risk of getting CSFVE.

Though available published data on higher CPE score and CSF escape are confusing and suggest the use of higher CPE score, ART is not associated with the neurocognitive benefit (Ellis et al. 2014). At the same time, published case reports and small series showed early and marked clinical benefit to optimizing ART to improve CNS penetration, as with our study (Canestri et al. 2010). CNS penetration and CPE score of ART could be an important factor for CSFVE as highlighted by one of our case from present series. One of our patient Mr. V M 32/M, on stable regimen TDF/FTC/IDV/r (5 pills/day) for 72 months with undetectable plasma HIV RNA with latest CD4 counts of $565 \mathrm{~mm}^{3}$, developed progressive tremors involving both hands, disturbing his daily work of holding, and inspecting diamonds after 6 months of changing ART to TDF/FTC/ATV/r (2 pills/day) to simplification to reduce pill burden. His CSF showed HIV RNA of 14,299 copies/ml. He had a rapid reversal of his symptoms on switching back to the original regimen (CPE score from 6 to 8 ).

The findings from our study emphasizes on the importance of clinicians being vigilant for recognizing clinical symptoms for making an early diagnosis of symptomatic CSFVE. 
Specifically, patients can have a gradual onset of isolated or combination of focal and nonfocal neurological symptoms with symptomatic CSFVE (Khoury et al. 2013). Several of our patients had one or more presenting symptoms like impaired memory, dizziness, tremors, and motor symptoms. Patient reported these symptoms during routine medical checkup as these interfered with their day-to-day activities and in some cases, patient's relativesinformed patients changed behavior, which patient was unaware of. Early diagnosis may assist in the prevention of further progressive neurological damage and disability like our five patients who had gait disturbances and two of our patients who presented late had bladder bowel incontinence.

Published reports suggested cognitive abnormalities were commonest presenting symptoms followed by ataxia and motor impairment (Cardenas et al. 2015). Similar to the previous findings in patients with symptomatic CSFVE, our study also reported elevated proteins and lymphocytic pleocytosis in CSF.

Differential viral resistance in plasma and CSF compartment can exist and could be a great concern to the clinician as a cause for CSFVE and its management. In our cohort, CSF GTR results were available for five patients and all had M184V mutation into CSF. Of these five patients, one was treatment-naive and one failed prior TDF/FTC/EFV and had M46I, N88S, and M184V mutations in to CSF; two patients failed AZT/3TC/NVP and had V106M, T215Y, Y181C, K219Q, M46I, V82A, and L90M mutations in to CSF while one with prior d4T/3TC/NVP failure had only M184V mutations. We had two patient's paired CSF and plasma GTR assay results, which did not show a different resistant pattern in two compartments but there are published case reports with different resistance profile in CSF and plasma playing a role in CSFVE, and such patients were benefited by adjusting ART according to GTR results (Fabbiani et al. 2015; Beguelin et al. 2016).

Prevailing HIV-1 clade in India is clade C. Conflicting information is available from studies looking at the association of HIV-1 clade and prevalence of neurocognitive abnormalities. Some studies report no association of HAND prevalence with different HIV-1 clades while other studies suggest clade D may be more neurovirulent followed by clades B, C, and A. Higher neurovirulence in clade $\mathrm{B}$ compared to clade $\mathrm{C}$ is related to difference in tat gene in clade B (Tyor et al. 2013).

The clinical response following treatment adjustment according to CPE score is debatable and still unclear (Cusini et al. 2013). Certain studies reported partial to no response and other reported rapid and early response in patients with symptomatic CSFVE after ART optimization (Canestri et al. 2010; Peluso et al. 2012; Khoury et al. 2013; Cardenas et al. 2015). We also noted overall quick clinical response after ART optimization. One patient with bladder and bowel disturbance with paraparesis regained bladder control in 8 days and started walking at 4 weeks without support. Three patients had a recurrence of same symptoms following non-adherence and virologically failing optimized ART. As this is a retrospective study and active search for patients with symptomatic CSF escape at our clinic was started from 2012 onwards; it is also possible that we might have missed few patients in our busy clinic with symptomatic CSF escape. These factors contribute to bias and underestimate actual incidence of symptomatic CSF escape. 
The study also has limitations, and one of them being the retrospective nature of the data which makes it difficult to account for confounders. However, given the paucity of evidence related to CSF escape associated with atazanavir-based regimens, this study is the first to assess such association and represents the largest cohort on this issue. That is, until a large prospective cohort study is performed, these findings are important to not only inform future studies but also inform clinicians to be observant while managing the disease. Furthermore, given the rarity CSF escape, a significant time lag is expected before a large prospective cohort study is completed, and therefore until then these findings represent the most comprehensive and largest study to date on the issue.

Symptomatic CSFVE with ATV/r containing regimen was a rare but clinically significant condition in this single-center study. Nadir CD4 count $\leq 200$ was associated with substantially increased risk of symptomatic CSFVE. Clinicians should be more vigilant for symptomatic CSFVE in patients with nadir CD4 count $<200$, further strengthening efforts to diagnose and treat patients early in the disease.

\section{References}

Antinori A, Clarke A, Svedhem-Johansson V, Arribas JR, Arenas-Pinto A, Fehr J, Gerstoft J, Horban A, Clotet B, Ripamonti D, Girard PM, Hill AM, Moecklinghoff C (2015) Week 48 efficacy and central nervous system analysis of darunavir/ritonavir monotherapy versus darunavir/ritonavir with two nucleoside analogues. AIDS 29(14): 1811-1820 [PubMed: 26372387]

Beguelin C, Vazquez M, Bertschi M, Yerly S, de Jong D, Gutbrod K, Rauch A, Cusini A (2016) Viral escape in the central nervous system with multidrug-resistant human immunodeficiency Virus-1. Open Forum Infect Dis 3(1):ofv210 [PubMed: 26885540]

Bogoch II, Davis BT, Venna N (2011) Reversible dementia in a patient with central nervous system escape of human immunodeficiency virus. J Inf Secur 63(3):236-239

Canestri A, Lescure FX, Jaureguiberry S, Moulignier A, Amiel C, Marcelin AG, Peytavin G, Tubiana R, Pialoux G, Katlama C (2010) Discordance between cerebral spinal fluid and plasma HIV replication in patients with neurological symptoms who are receiving suppressive antiretroviral therapy. Clin Infect Dis 50(5):773-778 [PubMed: 20100092]

Cardenas G, Lopez-Gonzalez M, Monzon-Falconi JF, Soto-Hernandez JL, Perales-Martinez D, LopezVejar C (2015) Relation of cerebro-spinal fluid/plasma HIV-RNA discordance with neurocognitive impairment. Natl Med J India 28(5):228-232 [PubMed: 27132951]

Christo PP, Greco DB, Aleixo AW, Livramento JA (2007) Factors influencing cerebrospinal fluid and plasma HIV-1 RNA detection rate in patients with and without opportunistic neurological disease during the HAART era. BMC Infect Dis 7:147 [PubMed: 18096083]

Cinque P, Vago L, Ceresa D, Mainini F, Terreni MR, Vagani A, Torri W, Bossolasco S, Lazzarin A (1998) Cerebrospinal fluid HIV-1 RNA levels: correlation with HIV encephalitis. AIDS 12(4):389394 [PubMed: 9520168]

Cusini A, Vernazza PL, Yerly S, Decosterd LA, Ledergerber B, Fux CA, Rohrbach J, Widmer N, Hirschel B, Gaudenz R, Cavassini M, Klimkait T, Zenger F, Gutmann C, Opravil M, Gunthard HF, Swiss HIVCS (2013) Higher CNS penetration-effectiveness of long-term combination antiretroviral therapy is associated with better HIV-1 viral suppression in cerebrospinal fluid. J Acquir Immune Defic Syndr 62(1):28-35 [PubMed: 23018371]

Donath M, Wolf T, Sturmer M, Herrmann E, Bickel M, Khaykin P, Gopel S, Gute P, Haberl A, de Leuw P, Schuttfort G, Berger A, Stephan C, H. I. V. C. S. for Frankfurt (2016) HIV-1 replication in central nervous system increases over time on only protease inhibitor therapy. Med Microbiol Immunol 205(6):575-583 [PubMed: 27469377]

Ellis RJ, Letendre S, Vaida F, Haubrich R, Heaton RK, Sacktor N, Clifford DB, Best BM, May S, Umlauf A, Cherner M, Sanders C, Ballard C, Simpson DM, Jay C, McCutchan JA (2014) 
Randomized trial of central nervous system-targeted antiretrovirals for HIV-associated neurocognitive disorder. Clin Infect Dis 58(7):1015-1022 [PubMed: 24352352]

Eden A, Fuchs D, Hagberg L, Nilsson S, Spudich S, Svennerholm B, Price RW, Gisslen M (2010) HIV-1 viral escape in cerebrospinal fluid of subjects on suppressive antiretroviral treatment. J Infect Dis 202(12):1819-1825 [PubMed: 21050119]

Eden A, Nilsson S, Hagberg L, Fuchs D, Zetterberg H, Svennerholm B, Gisslen M (2016) Asymptomatic cerebrospinal fluid HIV-1 viral blips and viral escape during antiretroviral therapy: a longitudinal study. J Infect Dis 214(12):1822-1825 [PubMed: 27683820]

Fabbiani M, Grima P, Milanini B, Mondi A, Baldonero E, Ciccarelli N, Cauda R, Silveri MC, De Luca A, Di Giambenedetto S (2015) Antiretroviral neuropenetration scores better correlate with cognitive performance of HIV-infected patients after accounting for drug susceptibility. Antivir Ther 20(4):441-447 [PubMed: 25516553]

Ferretti F, Gisslen M, Cinque P, Price RW (2015) Cerebrospinal fluid HIV escape from antiretroviral therapy. Curr HIV/AIDS Rep 12(2):280-288 [PubMed: 25860317]

Ferretti F, Bigoloni A, Passeri L, Galli L, Longo V, Gerevini S, Spagnuolo V, Gisslen M, Zetterberg H, Fuchs D, Cattaneo D, Caramatti G, Lazzarin A, Cinque P, Castagna A (2016) Cerebrospinal fluid analysis for HIV replication and biomarkers of immune activation and neurodegeneration in longterm atazanavir/ritonavir monotherapy treated patients. Medicine (Baltimore) 95(28):e4144 [PubMed: 27428202]

Khoury MN, Tan CS, Peaslee M, Koralnik IJ (2013) CSF viral escape in a patient with HIV-associated neurocognitive disorder. J Neuro-Oncol 19(4):402-405

Letendre SL, Ellis RJ, Ances BM, McCutchan JA (2010) Neurologic complications of HIV disease and their treatment. Top HIV Med 18(2):45-55 [PubMed: 20516524]

Nightingale S, Geretti AM, Beloukas A, Fisher M, Winston A, Else L, Nelson M, Taylor S, Ustianowski A, Ainsworth J, Gilson R, Haddow L, Ong E, Watson V, Leen C, Minton J, Post F, Pirmohamed M, Solomon T, Khoo S (2016) Discordant CSF/plasma HIV-1 RNA in patients with unexplained low-level viraemia. J Neuro-Oncol 22(6):852-860

Price RW, Spudich S (2008) Antiretroviral therapy and central nervous system HIV type 1 infection. J Infect Dis 197(Suppl 3):S294-S306 [PubMed: 18447615]

Peluso MJ, Ferretti F, Peterson J, Lee E, Fuchs D, Boschini A, Gisslen M, Angoff N, Price RW, Cinque P, Spudich S (2012) Cerebrospinal fluid HIVescape associated with progressive neurologic dysfunction in patients on antiretroviral therapy with well controlled plasma viral load. AIDS 26(14):1765-1774 [PubMed: 22614889]

Rawson T, Muir D, Mackie NE, Garvey LJ, Everitt A, Winston A (2012) Factors associated with cerebrospinal fluid HIV RNA in HIV infected subjects undergoing lumbar puncture examination in a clinical setting. J Inf Secur 65(3):239-245

Robertson K, Fiscus S, Kapoor C, Robertson W, Schneider G, Shepard R, Howe L, Silva S, Hall C (1998) CSF, plasma viral load and HIV associated dementia. J Neuro-Oncol 4(1):90-94

Santos JR, Munoz-Moreno JA, Molto J, Prats A, Curran A, Domingo P, Llibre JM, McClernon DR, Bravo I, Canet J, Watson V, Back D, Clotet B (2013) Virological efficacy in cerebrospinal fluid and neurocognitive status in patients with long-term monotherapy based on lopinavir/ritonavir: an exploratory study. PLoS One 8(7):e70201 [PubMed: 23922957]

Staprans S, Marlowe N, Glidden D, Novakovic-Agopian T, Grant RM, Heyes M, Aweeka F, Deeks S, Price RW (1999) Time course of cerebrospinal fluid responses to antiretroviral therapy: evidence for variable compartmentalization of infection. AIDS 13(9):1051-1061 [PubMed: 10397535]

Tamarit Mdel P, Quereda C, Gonzalez-Rozas M, Corral I, Casado JL (2012) HIV type 1 viral encephalitis after development of viral resistance to plasma suppressive antiretroviral therapy. AIDS Res Hum Retrovir 28(1):83-86 [PubMed: 21504362]

Tyor W, Fritz-French C, Nath A (2013) Effect of HIV clade differences on the onset and severity of HIV-associated neurocognitive disorders. J Neuro-Oncol 19(6):515-522

Vernazza P, Daneel S, Schiffer V, Decosterd L, Fierz W, Klimkait T, Hoffmann M, Hirschel B (2007) The role of compartment penetration in PI-monotherapy: the Atazanavir-Ritonavir Monomaintenance (ATARITMO) Trial. AIDS 21(10):1309-1315 [PubMed: 17545707] 
Yilmaz A, Svennerholm B, Hagberg L, Gisslen M (2006) Cerebrospinal fluid viral loads reach less than 2 copies/ml in HIV-1-infected patients with effective antiretroviral therapy. Antivir Ther 11(7):833-837 [PubMed: 17302245] 


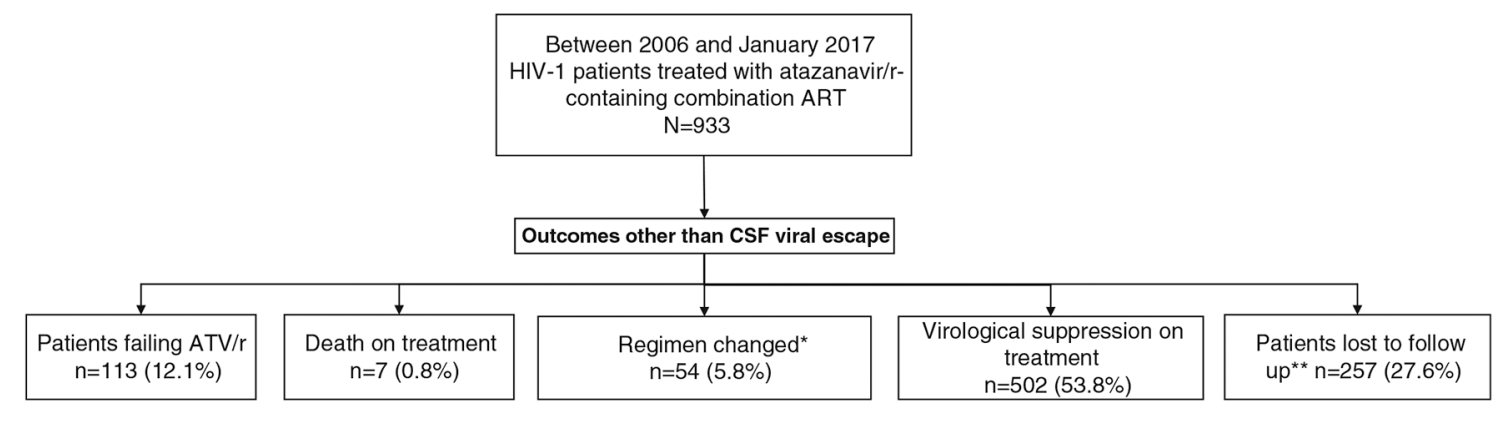

* Regimen changed due to adverse drug reactions, CSF viral escape

** Patients who received at least 3 months of treatment and lost to follow up

Fig. 1.

Cohort of patients receiving ATV/r based ART since 2006 


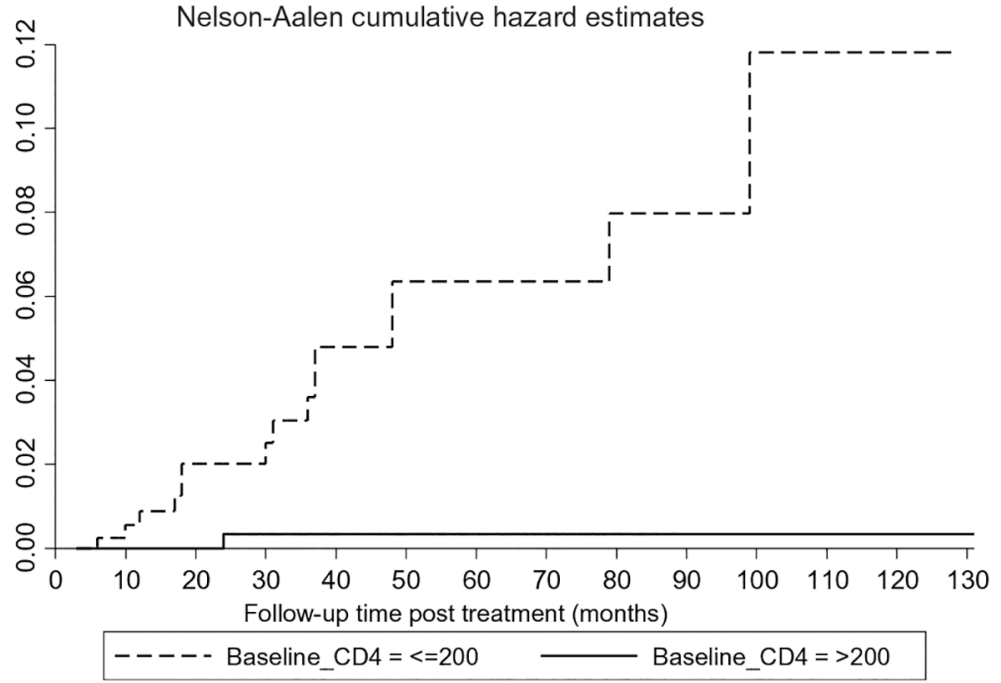

Fig. 2.

Cumulative incidence of CSF escapes in relation to baseline CD4 count after starting ATV/r containing treatment 


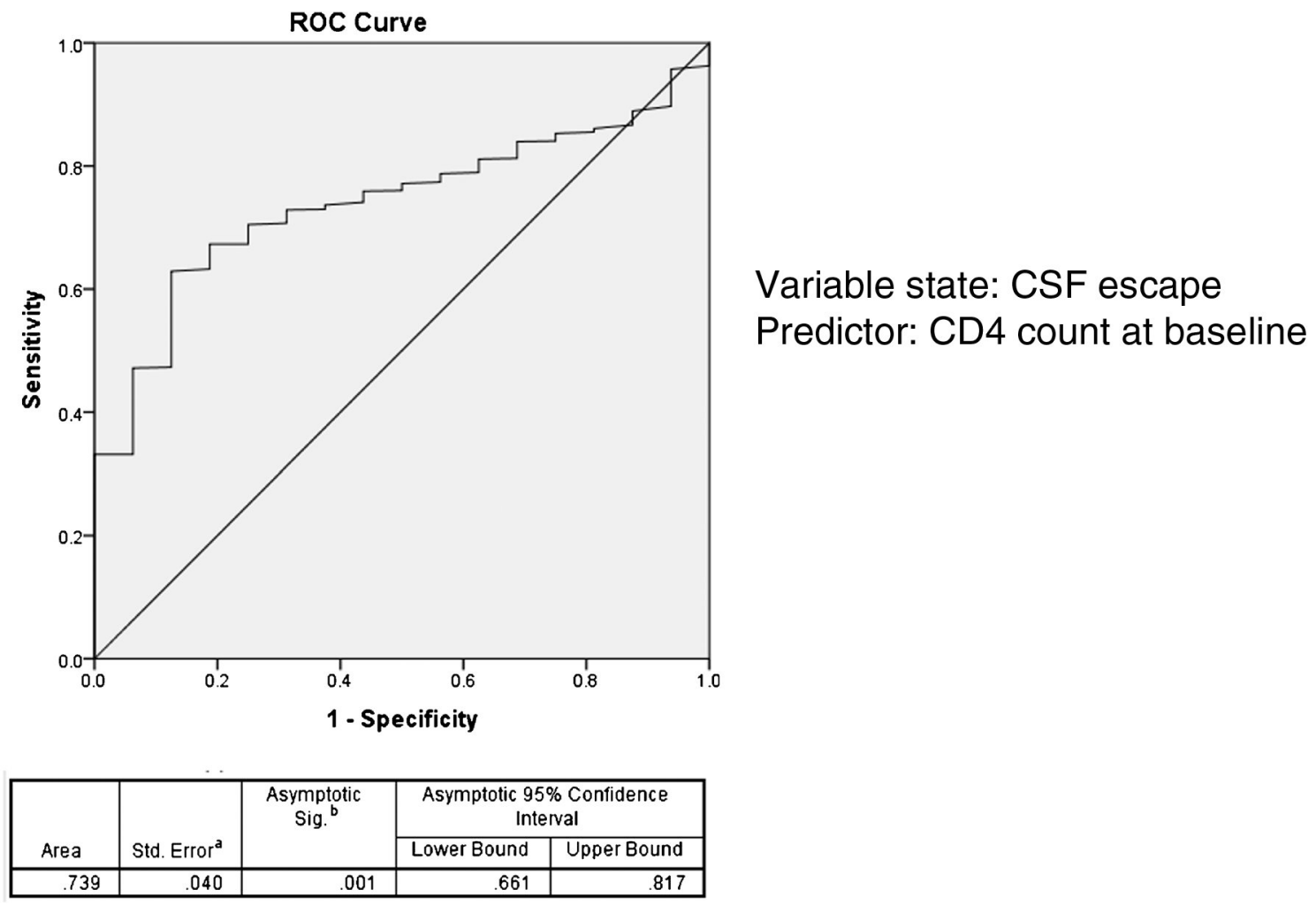

Fig. 3.

CSF escape Predictor: CD4 count at baseline 


\section{롤 \\ 일}

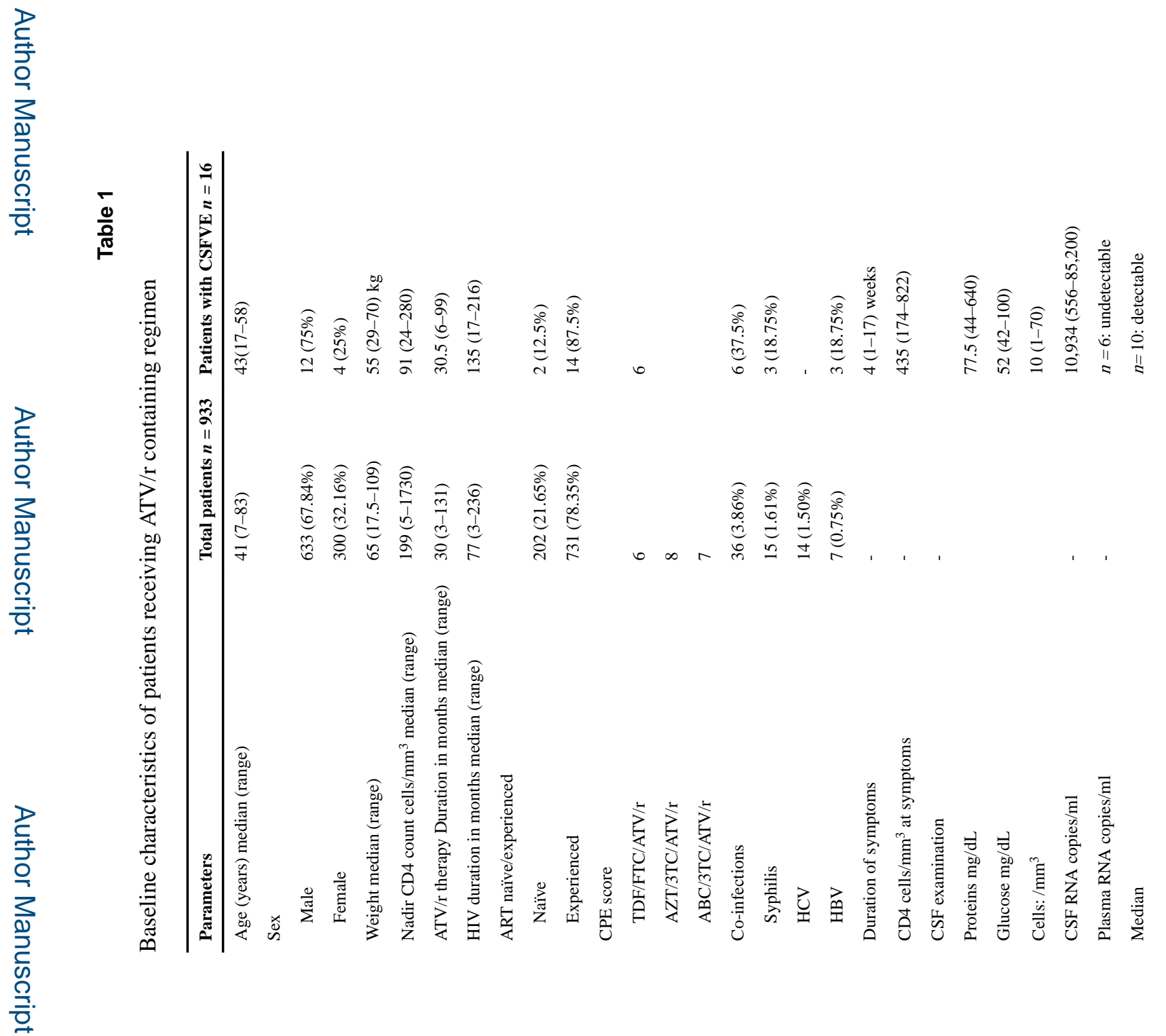

J Neurovirol. Author manuscript; available in PMC 2019 August 01. 
Patel et al.

Page 15

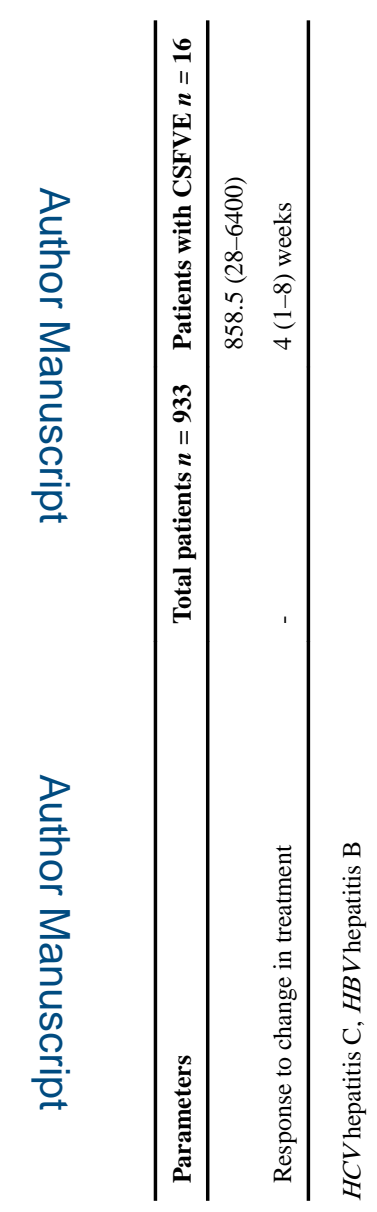

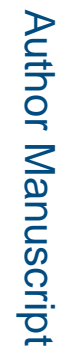

를

J Neurovirol. Author manuscript; available in PMC 2019 August 01. 


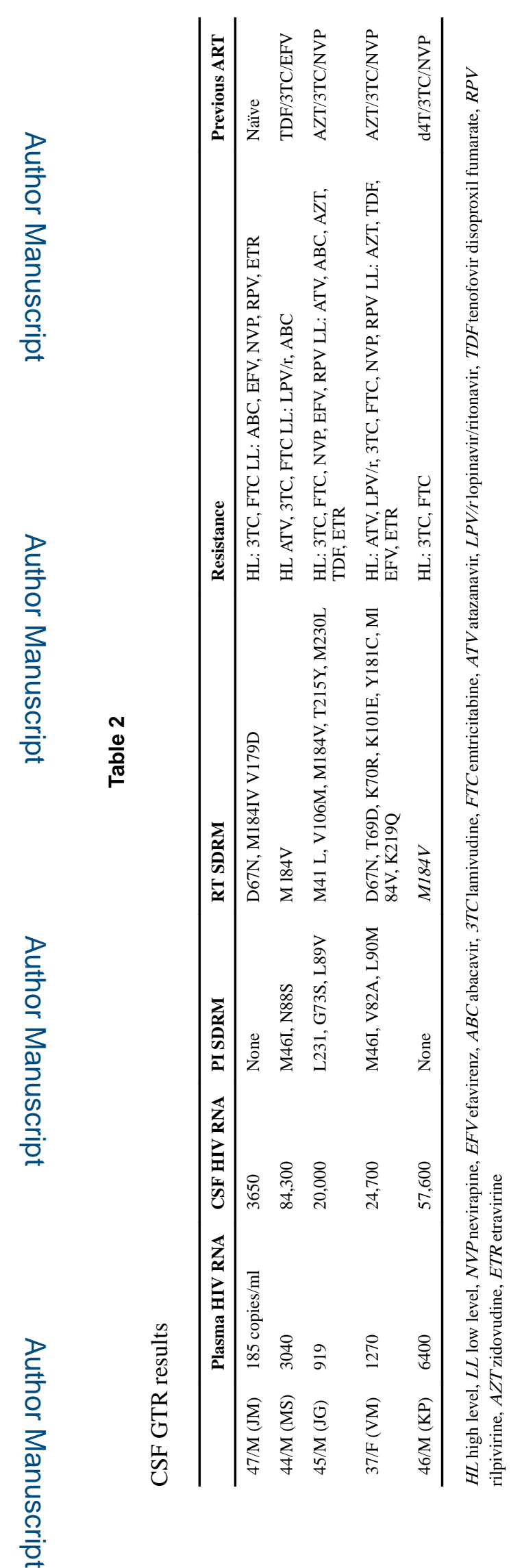

J Neurovirol. Author manuscript; available in PMC 2019 August 01. 


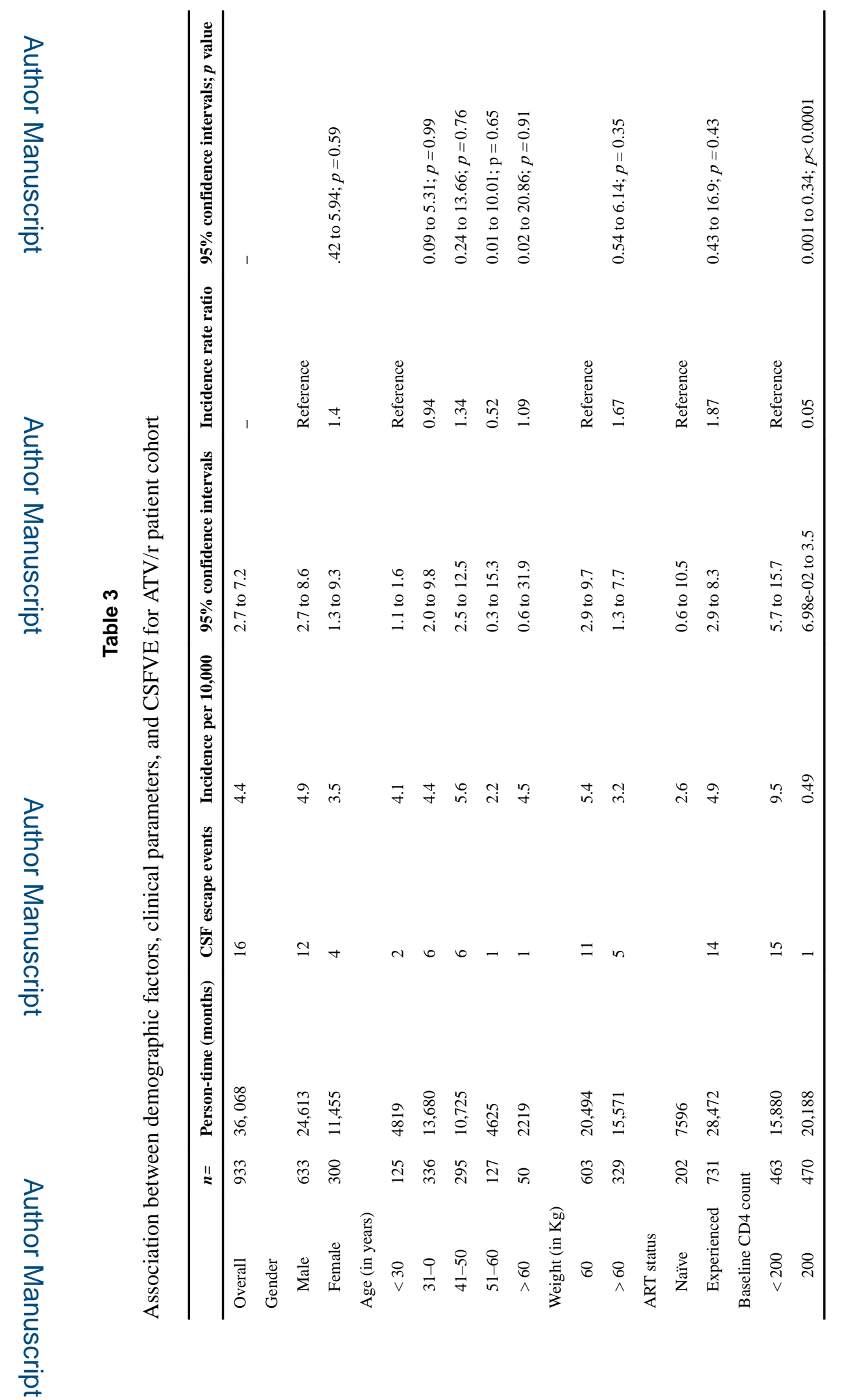

